\title{
Stream Sediments Geochemistry of the Nyambaka Drainage System Northern Cameroon (Central Africa): A Target for Mining Exploration
}

\author{
Edima Yana Roland William ${ }^{1,6, ~ *}$, Bidjo Emvoutou Gery Christian ${ }^{1,2}$, Okomo Atouba Lise Carole ${ }^{3}$, \\ Ondoua Oyono Joseph Sadrac ${ }^{4}$, Ipan Antoinette Solange ${ }^{1,5}$, Sep Nlomngan Jean Paul ${ }^{1,2}$, \\ Nkouandou Faarouk Oumarou ${ }^{6}$ \\ ${ }^{1}$ Centre for Geological and Mining Research, Garoua, Cameroon \\ ${ }^{2}$ Departement of Earth Sciences, University of Yaoundé I, Yaoundé, Cameroon \\ ${ }^{3}$ Higher Teacher Training College of Bertoua, University of Ngaoundere, Bertoua, Cameroon \\ ${ }^{4}$ Institute of Research for Agriculture Development, Division: Green Production, Yaoundé, Cameroon \\ ${ }^{5}$ Department of Earth Sciences, University of Yaoundé I, Yaoundé, Cameroon \\ ${ }^{6}$ Department of Earth Sciences, University of Ngaoundere, Ngaoundere, Cameroun \\ Email address: \\ goldrewy@yahoo.com (E. Y. R. William) \\ ${ }^{*}$ Corresponding author
}

\section{To cite this article:}

Edima Yana Roland William, Bidjo Emvoutou Gery Christian, Okomo Atouba Lise Carole, Ondoua Oyono Joseph Sadrac, Ipan Antoinette Solange, Sep Nlomngan Jean Paul, Nkouandou Faarouk Oumarou. Stream Sediments Geochemistry of the Nyambaka Drainage System Northern Cameroon (Central Africa): A Target for Mining Exploration. International Journal of Statistical Distributions and Applications. Vol. 7, No. 2, 2021, pp. 25-34. doi: 10.11648/j.jjsd.20210702.11

Received: April 27, 2021; Accepted: May 15, 2021; Published: June 7, 2021

\begin{abstract}
Mining exploration in the Nyambaka area Northern Cameroon still at reconnaissance stage. In this study, ten active stream sediments samples were collected for geochemical survey. These stream sediments were analyzed by inductively coupled Plasma/ Mass Spectometry (ICP/MS), the data set obtained was transformed into a standard formation an excel database, and was subject to statistical treatment using IBM SPSS statistics 21 for 33 chemical elements to highlight the relationship between the stream sediments geochemistry, the region lithology, the geological processes and eventual primary mineralization. The data were analyzed using multivariate statistics. R-mode analysis produced a five-factor model behind multi-elements associations which account for $93.40 \%$ of the total variance in the data with the following metals associations: Sc-Mo-V-In-Ga-Cu-Cr, Ba-Sr-Ag-Cr-Cu-Co-Be-Ni-Y-Zn-V, Ga-Hf-Zr, Sn-Au, As-Cd. Sn-Au association indicates that Au mineralization is link to $\mathrm{Sn}$ mineralization. As- $\mathrm{Cd}, \mathrm{Cu} \mathrm{Ga}$, In, Mo suggested that the paragenesis represent others sulphidation events that is barren with respect to Au. The spatial distribution of Sn-Au and As-Cd factors show that these factors are mores express in the centre part of the study area; they can be link to granitic rocks, defining a primary gold target. A detail mining investigation have to me carry out in that area to highlight the primary mineralizations.
\end{abstract}

Keywords: Stream Sediments, Nyambaka Area, Northern Cameroon

\section{Introduction}

Geochemical mapping surveys have been conducted in different parts of the word at various scales to booster geochemical prospecting [1]. Acceptance of sediments composition is representative of the geochemistry of the catchment basin upstream [2]. The spatial display of stream sediment geochemical data, and statistical treatment of the data can unravel element associations relevant to primary exploration in a region [3], and high mining potential area. these associations are useful in speculating on the source region lithology, geological processes and the nature of the primary (rock-hosted) mineralization, if any is present $[4,5$, $1]$. 
The study area is a part of the Adamawa-Yadé domain, and was subject to geochemical, petrological, and geochronological studies of the basement rocks [6-9]. A few is known about mining index in the Adamawa- Yadé; and the study area is not yet subject to a geochemical exploration of stream sediments. The aim of this study is to do the mapping of the distribution of chemical elements in the study area watershed, to identify area of high mining potential for the boosting of mining exploration.

\section{Geographical and Geological Setting}

The study area is located in the Adamawa region, and lies between longitudes $10^{\circ} 0^{\prime} \mathrm{E}$ and $14^{\circ} 09^{\prime} \mathrm{E}$, and latitudes $6^{\circ} 60^{\prime} \mathrm{N}$ and $6^{\circ} 52^{\prime} \mathrm{N}$ respectively. It's hydrographic system belong to two main hydrographic basins known in Cameroon the Tchad Basin and the Sanaga Basin (Olivry, 1986) (figure 1A); and to the Mayo Mambaka Wathersheed (figure 1B).

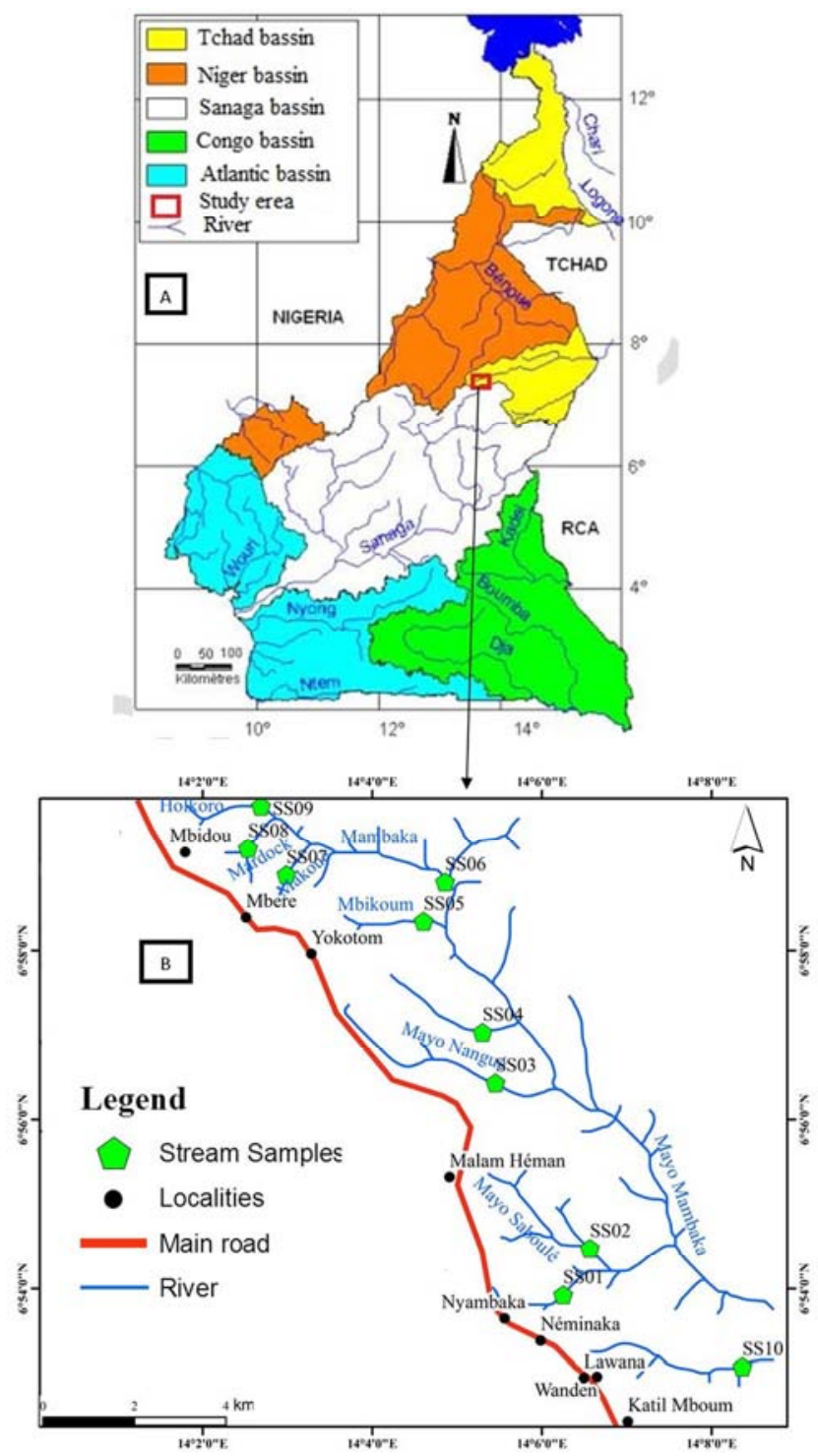

Figure 1. Maps: A- Hydrographic bassins of Cameroon (Olivry, 1986); BThe Mayo Mambaka Wathersheed and Stream sediment sampling.

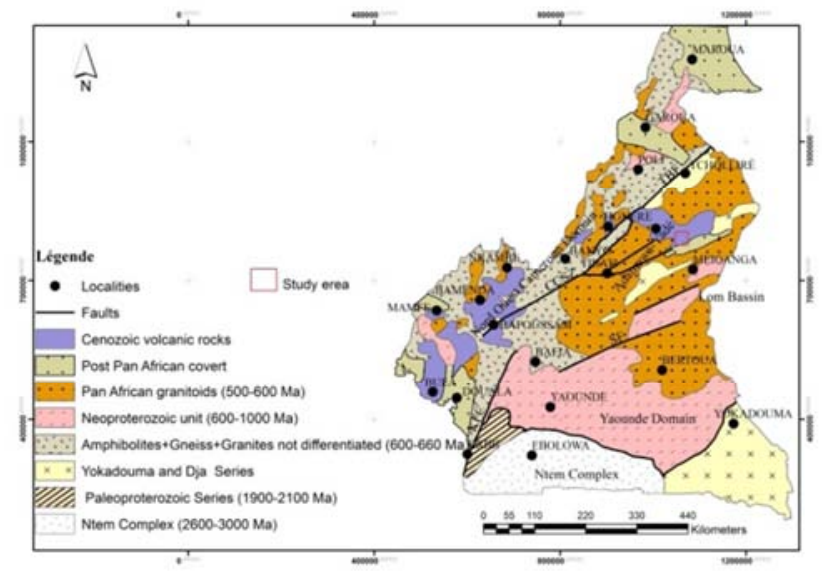

Figure 2. Geological map of the Central African Orogenic Belt (CAOB) in Cameroon.

Geologically, the study area belongs to the Adamawa-Yadé domain, which is a part of the Central Africa, Orogenic Belt (CAOB) [10]. In Cameroon, the Central African Orogenic Belt $(\mathrm{CAOB})$ is subdivided into three geological domains, from the North to the South (figure 2): the Northern domain, the Adamawa-Yadé Domain, the Southern nappes (figure 2). The Adamawa-Yadé domain is made up of various low-to high-grade metamorphic rocks and abundant pan-african granites, bearing Archaean to palaeoproterozoïc isotopic signatures [11, 12]. Reference [13] suggest that the Adamaoua-Yadé domain represents an Archaean/palaeoproterozoîc microcontinent, which was detached from the northern margin of the Congo Craton in the early Neoproterozoïc, but became reaccreted together with the Mayo Kebbi magmatic arc during the Pan-African orogeny.

\section{Material and Methods}

Ten active stream sediments samples were collected within the drainage system (figure 1B). First and second tributaries rivers were sampled. These samples were weighed with an electronic balance (Sartorius et Kitchen scale SF-400), and air dry in the "Laboratory of Mineral Treatment"; after drying, samples were crushed using a "Fritsch Pulverizer", and $30 \mathrm{~g}$ of $180 \mu \mathrm{m}$ fraction was retained for further geochemical analysis.

In the laboratory, a $0.2 \mathrm{~g}$ of each sample was analyzed for 65 chemical elements by inductively coupled Plasma/ Mass Spectometry (ICP/MS) at 'Bureau Veritas Laboratories Canada". Replicates samples chosen from the sample batch were randomly place in each analysis to test for analytical precision.

The data set obtained was transformed into a standard formation an excel database, and was subject to statistical treatment using IBM SPSS statistics 21 for 33 chemical elements (table 6). Statistical parameters like minimum, median, mean, maximum were measured to have central tendency; while standard deviation, variance, give statistical dispersion. The asymmetry is given by Skewness and 
Kurtosis index. Correlation matrix, factor analysis were performed to obtain trends and relationship between variables, and deduce the factors influencing the stream sediment geochemistry.

Colored geochemical maps of the data were draw using ArcGis 10.2 software. Symbol plots of factor scores of the elements associations obtained after factor analysis, were produced to analyze their relationship with the geological catchment of the area. These factor scores were projected on a map along the watershed system to present sectors of high mining potentials.

\section{Results and Discussion}

Ten samples were analyzed for a whole suite of elements; only 33 elements with concentrations above the detection limit are reported (table 1). The descriptive statistics are presented in table 2. Twenty elements present positive skewed distribution.

Table 1. Detections limits of 33 elements concentrations.

\begin{tabular}{|c|c|c|c|c|c|c|c|c|}
\hline Elements & Unit & DL & Elements & Unit & DL & Elements & Unit & DL \\
\hline Mo & ppm & 0.01 & $\mathrm{Cd}$ & ppm & 0.01 & Cs & Ppm & 0.02 \\
\hline $\mathrm{Cu}$ & ppm & 0.01 & $\mathrm{Sb}$ & ppm & 0.02 & Hf & Ppm & 0.02 \\
\hline $\mathrm{Pb}$ & ppm & 0.01 & $\mathrm{Bi}$ & ppm & 0.02 & $\mathrm{Nb}$ & Ppm & 0.02 \\
\hline $\mathrm{Zn}$ & ppm & 0.1 & $\mathrm{~V}$ & ppm & 1 & $\mathrm{Rb}$ & Ppm & 0.1 \\
\hline $\mathrm{Ag}$ & $\mathrm{ppb}$ & 2 & $\mathrm{Ca}$ & $\%$ & 0.01 & $\mathrm{Sn}$ & Ppm & 0.1 \\
\hline $\mathrm{Ni}$ & ppm & 0.1 & $\mathrm{Cr}$ & ppm & 0.5 & $\mathrm{Zr}$ & Ppm & 0.1 \\
\hline $\mathrm{Co}$ & ppm & 0.1 & $\mathrm{Ba}$ & ppm & 0.5 & $\mathrm{Y}$ & Ppm & 0.01 \\
\hline $\mathrm{U}$ & ppm & 0.1 & $\mathrm{Tl}$ & ppm & 0.02 & $\mathrm{Be}$ & Ppm & 0.1 \\
\hline $\mathrm{Au}$ & $\mathrm{ppb}$ & 0.2 & $\mathrm{Hg}$ & $\mathrm{ppb}$ & 5 & $\mathrm{Sr}$ & Ppm & 0.5 \\
\hline Th & ppm & 0.1 & $\mathrm{Se}$ & ppm & 0.1 & $\mathrm{Ga}$ & Ppm & 0.1 \\
\hline
\end{tabular}

Table 2. The descriptive statistics $(N=10)$.

\begin{tabular}{|c|c|c|c|c|c|c|c|c|}
\hline & Minimum & Maximum & Mean & Variance & Skewness & & Kurtosis & \\
\hline & Statistic & Statistic & Statistic & Statistic & Statistic & Erreur std & Statistic & Erreur std \\
\hline Mo & 1.42 & 2.06 & 1.77 & 0.042 & -0.51 & 0.68 & -0.692 & 1.33 \\
\hline $\mathrm{Cu}$ & 22.54 & 54.21 & 41.12 & 143.43 & -0.67 & 0.68 & -1.19 & 1.33 \\
\hline $\mathrm{Pb}$ & 5.81 & 13.31 & 8.24 & 6.98 & 1.24 & 0.68 & 0.47 & 1.33 \\
\hline $\mathrm{Zn}$ & 29.20 & 89.60 & 62.23 & 385.62 & -0.20 & 0.68 & -0.98 & 1.33 \\
\hline $\mathrm{Ag}$ & 15.00 & 24.00 & 20.5 & 9.61 & -0.89 & 0.68 & -0.55 & 1.33 \\
\hline $\mathrm{Ni}$ & 36.40 & 231.10 & 134.29 & 3615.35 & -0.00 & 0.68 & -0.74 & 1.33 \\
\hline As & 1.20 & 2.60 & 1.47 & 0.17 & 2.70 & 0.68 & 7.92 & 1.33 \\
\hline $\mathrm{U}$ & 2.20 & 4.00 & 2.69 & 0.42 & 1.43 & 0.68 & 0.82 & 1.33 \\
\hline $\mathrm{Au}$ & 0.40 & 1.00 & 0.79 & 0.04 & -0.63 & 0.68 & -0.34 & 1.33 \\
\hline $\mathrm{Th}$ & 9.80 & 25.60 & 15.19 & 21.31 & 1.47 & 0.68 & 2.11 & 1.33 \\
\hline $\mathrm{Sr}$ & 7.10 & 105.00 & 32.36 & 764.96 & 2.32 & 0.68 & 6.27 & 1.33 \\
\hline $\mathrm{Cd}$ & 0.01 & 0.06 & 0.03 & 0.00 & 0.19 & 0.68 & -0.14 & 1.33 \\
\hline $\mathrm{Sb}$ & 0.00 & 0.06 & 0.02 & 0.00 & 0.03 & 0.68 & -1.22 & 1.33 \\
\hline $\mathrm{Ca}$ & 0.00 & 0.25 & 0.07 & 0.00 & 1.78 & 0.68 & 3.49 & 1.33 \\
\hline $\mathrm{Cr}$ & 202.70 & 763.00 & 443.20 & 32084.82 & 0.23 & 0.68 & -0.51 & 1.33 \\
\hline $\mathrm{Ba}$ & 43.20 & 325.20 & 134.45 & 5997.41 & 1.74 & 0.68 & 4.26 & 1.33 \\
\hline $\mathrm{Sc}$ & 15.20 & 34.50 & 28.23 & 39.44 & -1.28 & 0.68 & 0.74 & 1.33 \\
\hline $\mathrm{Tl}$ & 0.05 & 0.22 & 0.09 & 0.00 & 1.69 & 0.68 & 1.25 & 1.33 \\
\hline $\mathrm{Hg}$ & 17.00 & 54.00 & 26.40 & 121.15 & 1.95 & 0.68 & 4.61 & 1.33 \\
\hline $\mathrm{Se}$ & 0.00 & 0.20 & 0.08 & 0.00 & 0.13 & 0.68 & 0.17 & 1.33 \\
\hline $\mathrm{Ga}$ & 20.40 & 34.60 & 27.50 & 17.93 & 0.05 & 0.68 & -0.36 & 1.33 \\
\hline Cs & 0.44 & 1.12 & 0.73 & 0.06 & 0.44 & 0.68 & -1.36 & 1.33 \\
\hline Hf & 0.34 & 0.89 & 0.60 & 0.03 & 0.00 & 0.68 & -1.61 & 1.33 \\
\hline $\mathrm{Nb}$ & 0.91 & 4.04 & 1.92 & 0.89 & 1.29 & 0.68 & 1.76 & 1.33 \\
\hline $\mathrm{Rb}$ & 2.30 & 18.1 & 5.94 & 38.12 & 1.75 & 0.68 & 1.37 & 1.33 \\
\hline $\mathrm{Sn}$ & 2.80 & 3.80 & 3.08 & 0.08 & 1.75 & 0.68 & 3.98 & 1.33 \\
\hline $\mathrm{Zr}$ & 27.00 & 61.50 & 42.86 & 158.32 & 0.16 & 0.68 & -1.41 & 1.33 \\
\hline $\mathrm{Y}$ & 12.20 & 27.88 & 23.15 & 24.69 & -1.21 & 0.68 & 1.43 & 1.33 \\
\hline
\end{tabular}

Elements relationship was investigate using a Pearson's correlation matrix (table 3) the correlation values (r) display a wide range from -0.4 between $\mathrm{Au}$ and $\mathrm{Se}$, to 0.9 between $\mathrm{Au}$ and $\mathrm{U}$, and between $\mathrm{Au}$ and $\mathrm{Zn}$. other elements pairs with a high positive $r$ values ( $\geq 0.9$ ) include $\mathrm{V}-\mathrm{Cr}$, Mo-Ni, Ni-Zn, U-Th, Sc-V (table 3). 
Table 3. $(a, b, c)$. Pearson's correlation matrix $(N=10)$.

\begin{tabular}{|c|c|c|c|c|c|c|c|c|c|c|}
\hline a) & Mo & $\mathrm{Cu}$ & $\mathbf{P b}$ & $\mathbf{Z n}$ & Ag & $\mathrm{Ni}$ & Co & As & $\mathbf{U}$ & Au \\
\hline Mo & 1.00 & & & & & & & & & \\
\hline $\mathrm{Cu}$ & 0.28 & 1.00 & & & & & & & & \\
\hline $\mathrm{Pb}$ & -0.63 & -0.77 & 1.00 & & & & & & & \\
\hline $\mathrm{Zn}$ & -0.00 & 0.88 & -0.59 & 1.00 & & & & & & \\
\hline $\mathrm{Ag}$ & 0.23 & 0.68 & -0.48 & 0.59 & 1.00 & & & & & \\
\hline $\mathrm{Ni}$ & 0.04 & 0.92 & -0.65 & 0.97 & 0.61 & 1.00 & & & & \\
\hline $\mathrm{Co}$ & -0.21 & 0.71 & -0.45 & 0.83 & 0.68 & 0.84 & 1.00 & & & \\
\hline As & 0.35 & 0.01 & -0.00 & -0.09 & 0.10 & -0.17 & -0.34 & 1.00 & & \\
\hline $\mathrm{U}$ & -0.76 & -0.63 & 0.96 & -0.44 & -0.36 & -0.50 & -0.27 & -0.03 & 1.00 & \\
\hline $\mathrm{Au}$ & 0.27 & 0.23 & -0.05 & -0.01 & 0.07 & -0.03 & -0.10 & 0.38 & -0.00 & 1.00 \\
\hline Th & -0.74 & -0.48 & 0.89 & -0.29 & -0.34 & -0.33 & -0.16 & -0.22 & 0.94 & 0.02 \\
\hline $\mathrm{Sr}$ & -0.35 & 0.53 & -0.34 & 0.63 & 0.34 & 0.58 & 0.80 & -0.24 & -0.15 & 0.18 \\
\hline $\mathrm{Cd}$ & 0.10 & 0.32 & -0.26 & 0.425 & 0.15 & 0.27 & 0.21 & 0.62 & -0.23 & 0.36 \\
\hline $\mathrm{Sb}$ & 0.48 & -0.53 & 0.24 & -0.71 & -0.42 & -0.72 & -0.83 & 0.54 & 0.04 & 0.30 \\
\hline $\mathrm{Bi}$ & 0.15 & -0.67 & 0.55 & -0.88 & -0.43 & -0.87 & -0.84 & 0.32 & 0.42 & 0.40 \\
\hline V & 0.57 & 0.88 & -0.81 & 0.68 & 0.54 & 0.75 & 0.414 & 0.14 & -0.77 & 0.22 \\
\hline $\mathrm{Cr}$ & 0.27 & 0.87 & -0.75 & 0.77 & 0.49 & 0.86 & 0.57 & -0.17 & -0.67 & -0.09 \\
\hline $\mathrm{Ba}$ & -0.49 & 0.44 & -0.16 & 0.62 & 0.29 & 0.55 & 0.79 & -0.27 & 0.02 & 0.14 \\
\hline $\mathrm{Sc}$ & 0.61 & 0.82 & -0.83 & 0.52 & 0.54 & 0.61 & 0.36 & 0.06 & -0.79 & 0.37 \\
\hline $\mathrm{Tl}$ & -0.76 & -0.68 & 0.93 & -0.41 & -0.29 & -0.49 & -0.19 & -0.10 & 0.95 & -0.23 \\
\hline $\mathrm{Hg}$ & 0.40 & -0.41 & -0.19 & -0.47 & -0.27 & -0.51 & -0.42 & 0.27 & -0.32 & -0.14 \\
\hline $\mathrm{Se}$ & 0.50 & -0.24 & -0.24 & -0.18 & -0.05 & -0.23 & -0.34 & 0.27 & -0.43 & -0.45 \\
\hline $\mathrm{Ga}$ & 0.78 & 0.24 & -0.39 & -0.16 & 0.20 & -0.07 & -0.31 & 0.36 & -0.48 & 0.49 \\
\hline Cs & -0.49 & -0.82 & 0.90 & -0.77 & -0.42 & -0.81 & -0.52 & 0.12 & 0.86 & 0.07 \\
\hline $\mathrm{Hf}$ & 0.51 & 0.25 & -0.07 & 0.13 & 0.26 & 0.16 & -0.11 & 0.23 & -0.18 & 0.29 \\
\hline $\mathrm{Nb}$ & 0.29 & -0.66 & 0.16 & -0.58 & -0.37 & -0.66 & -0.58 & 0.34 & -0.04 & -0.24 \\
\hline $\mathrm{Rb}$ & -0.83 & -0.64 & 0.92 & -0.35 & -0.34 & -0.44 & -0.15 & -0.15 & 0.95 & -0.19 \\
\hline $\mathrm{Sn}$ & 0.32 & 0.07 & 0.05 & -0.31 & 0.01 & -0.18 & -0.32 & 0.04 & 0.05 & 0.61 \\
\hline $\mathrm{Zr}$ & 0.6 & 0.41 & -0.26 & 0.27 & 0.39 & 0.31 & 0.02 & 0.16 & -0.35 & 0.28 \\
\hline Y & -0.20 & 0.70 & -0.27 & 0.82 & 0.74 & 0.78 & 0.85 & -0.23 & -0.09 & 0.08 \\
\hline In & 0.70 & 0.68 & -0.69 & 0.32 & 0.56 & 0.41 & 0.15 & 0.14 & -0.67 & 0.44 \\
\hline $\mathrm{Be}$ & -0.20 & 0.63 & -0.17 & 0.80 & 0.482 & 0.74 & 0.55 & 0.23 & -0.03 & -0.06 \\
\hline $\mathrm{Ca}$ & -0.55 & 0.26 & -0.00 & 0.42 & 0.25 & 0.34 & 0.67 & -0.23 & 0.17 & 0.21 \\
\hline
\end{tabular}

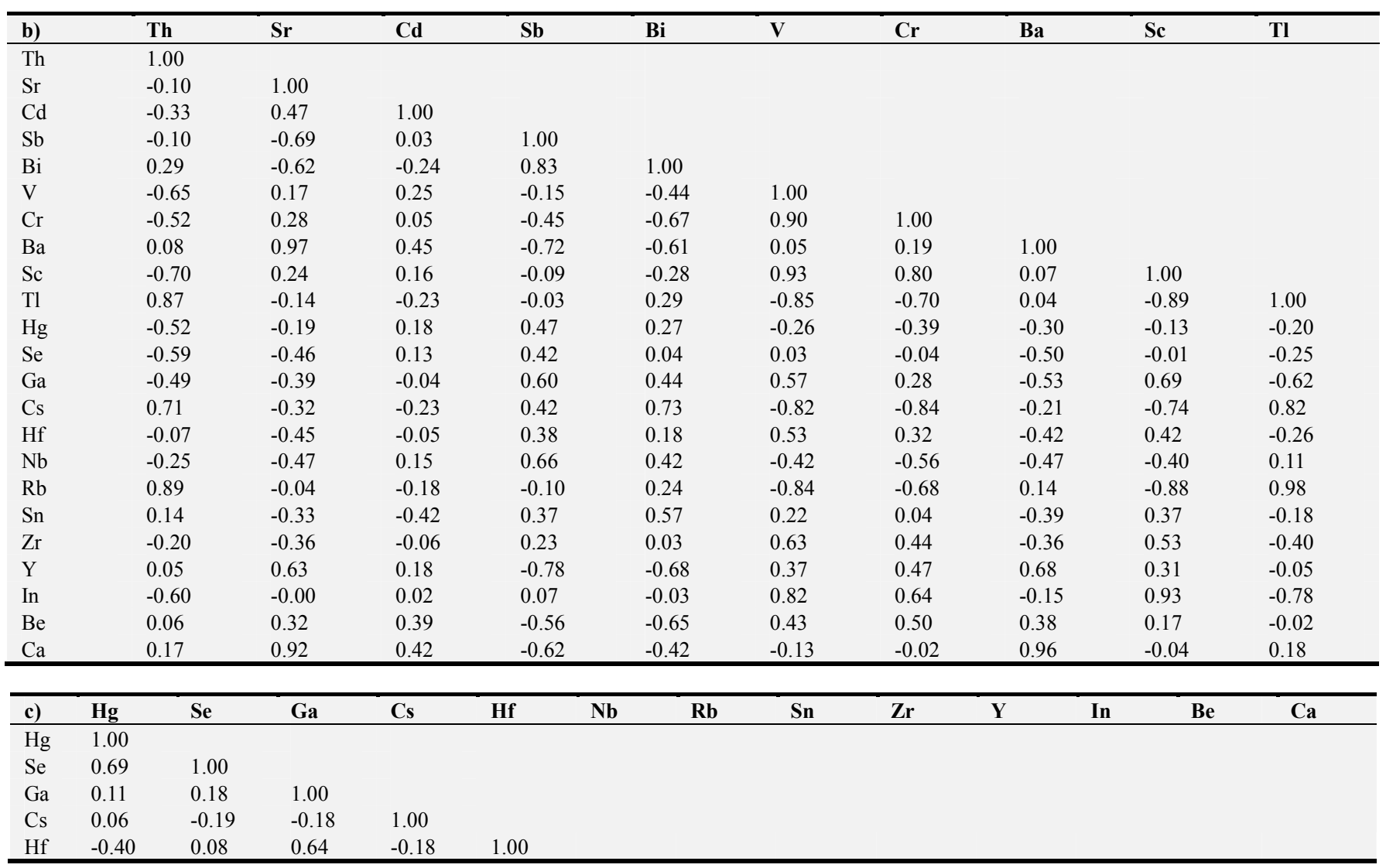




\begin{tabular}{|c|c|c|c|c|c|c|c|c|c|c|c|c|c|}
\hline c) & $\mathrm{Hg}$ & Se & $\mathbf{G a}$ & Cs & Hf & $\mathbf{N b}$ & $\mathbf{R b}$ & Sn & $\mathrm{Zr}$ & $\mathbf{Y}$ & In & $\mathrm{Be}$ & $\mathrm{Ca}$ \\
\hline $\mathrm{Nb}$ & 0.82 & 0.81 & 0.05 & 0.29 & -0.09 & 1.00 & & & & & & & \\
\hline $\mathrm{Rb}$ & -0.23 & -0.31 & -0.68 & 0.78 & -0.32 & 0.05 & 1.00 & & & & & & \\
\hline $\mathrm{Sn}$ & -0.29 & -0.44 & 0.70 & 0.16 & 0.49 & -0.36 & -0.21 & 1.00 & & & & & \\
\hline $\mathrm{Zr}$ & -0.39 & 0.09 & 0.63 & -0.38 & 0.96 & -0.17 & -0.46 & 0.46 & 1.00 & & & & \\
\hline Y & -0.63 & -0.41 & -0.26 & -0.44 & 0.09 & -0.67 & -0.01 & -0.15 & 0.23 & 1.00 & & & \\
\hline In & -0.12 & 0.03 & 0.82 & -0.58 & 0.55 & -0.32 & -0.80 & 0.54 & 0.65 & 0.24 & 1.00 & & \\
\hline $\mathrm{Be}$ & -0.59 & -0.23 & -0.28 & -0.41 & 0.17 & -0.53 & -0.00 & -0.27 & 0.24 & 0.69 & 0.07 & 1.00 & \\
\hline $\mathrm{Ca}$ & -0.21 & -0.51 & -0.52 & -0.00 & -0.50 & -0.36 & 0.28 & -0.35 & -0.47 & 0.59 & -0.22 & 0.23 & 1.00 \\
\hline
\end{tabular}

Factor analysis was applied on the log-transformed geochemical data to infer the controlling factors behind multi-elements associations in relation to catchment geology and mineralizations. The factor analysis using the varimax normalization Kaiser Method produced a five-factor model accounting for $93.40 \%$ (table 4 ) of the total data variance. The spatial distribution of these factors whiting the drainage system is presented in figure 3 . These factors are presented as follows (table 4):

Table 4. Factor analysis with varimax rotation for 33 trace metals in stream sediments $(N=10)$.

\begin{tabular}{|c|c|c|c|c|c|}
\hline \multirow{2}{*}{ Variables } & \multicolumn{5}{|c|}{ Factors } \\
\hline & 1 & 2 & 3 & 4 & 5 \\
\hline Mo & 0.79 & -0.29 & 0.39 & -0.00 & 0.19 \\
\hline $\mathrm{Cu}$ & 0.60 & 0.75 & 0.06 & 0.20 & 0.07 \\
\hline $\mathrm{Pb}$ & -0.93 & -0.30 & 0.11 & 0.08 & -0.02 \\
\hline $\mathrm{Zn}$ & 0.32 & 0.92 & -0.02 & -0.08 & 0.12 \\
\hline $\mathrm{Ag}$ & 0.33 & 0.60 & 0.18 & 0.07 & 0.14 \\
\hline $\mathrm{Ni}$ & 0.40 & 0.90 & 0.01 & -0.02 & -0.03 \\
\hline $\mathrm{Co}$ & 0.17 & 0.87 & -0.28 & -0.00 & -0.07 \\
\hline As & 0.06 & -0.19 & 0.28 & 0.02 & 0.86 \\
\hline $\mathrm{U}$ & -0.97 & -0.11 & 0.00 & 0.18 & -0.03 \\
\hline $\mathrm{Au}$ & 0.14 & -0.06 & 0.04 & 0.84 & 0.44 \\
\hline $\mathrm{Th}$ & -0.93 & 0.04 & 0.07 & 0.25 & -0.19 \\
\hline $\mathrm{Sr}$ & 0.10 & 0.62 & -0.70 & 0.22 & 0.12 \\
\hline $\mathrm{Cd}$ & 0.16 & 0.24 & -0.25 & -0.04 & 0.89 \\
\hline $\mathrm{Sb}$ & 0.06 & -0.82 & 0.38 & 0.08 & 0.33 \\
\hline $\mathrm{Bi}$ & -0.25 & -0.83 & 0.25 & 0.39 & 0.11 \\
\hline $\mathrm{V}$ & 0.77 & 0.46 & 0.34 & 0.13 & 0.07 \\
\hline $\mathrm{Cr}$ & 0.63 & 0.63 & 0.21 & -0.01 & -0.21 \\
\hline $\mathrm{Ba}$ & -0.09 & 0.68 & -0.64 & 0.18 & 0.13 \\
\hline $\mathrm{Sc}$ & 0.85 & 0.30 & 0.18 & 0.33 & 0.00 \\
\hline $\mathrm{Tl}$ & -0.97 & -0.09 & -0.04 & -0.07 & -0.04 \\
\hline $\mathrm{Hg}$ & 0.33 & -0.69 & -0.38 & -0.39 & 0.25 \\
\hline $\mathrm{Se}$ & 0.36 & -0.41 & 0.16 & -0.75 & 0.20 \\
\hline $\mathrm{Ga}$ & 0.63 & -0.34 & 0.51 & 0.41 & 0.10 \\
\hline Cs & -0.77 & -0.52 & -0.03 & 0.20 & 0.06 \\
\hline Hf & 0.19 & 0.08 & 0.89 & 0.18 & 0.10 \\
\hline $\mathrm{Nb}$ & 0.00 & -0.72 & -0.03 & -0.54 & 0.33 \\
\hline $\mathrm{Rb}$ & -0.98 & -0.04 & -0.13 & -0.04 & -0.04 \\
\hline $\mathrm{Sn}$ & 0.13 & -0.26 & 0.44 & 0.79 & -0.23 \\
\hline $\mathrm{Zr}$ & 0.34 & 0.18 & 0.85 & 0.15 & 0.04 \\
\hline $\mathrm{Y}$ & 0.00 & 0.91 & -0.01 & 0.13 & 0.00 \\
\hline In & 0.77 & 0.14 & 0.38 & 0.39 & 0.00 \\
\hline $\mathrm{Be}$ & 0.09 & 0.85 & 0.23 & -0.13 & 0.29 \\
\hline $\mathrm{Ca}$ & -0.18 & 0.40 & -0.84 & 0.17 & 0.13 \\
\hline
\end{tabular}

Factor 1: account for $39.20 \%$ of the total variability, and show high positive loadings for (tab.) Sc-Mo-V-In-Ga-Cu-Cr. these elements association can be interpreted to reflect an hydrothermal alteration leading to the development of $\mathrm{Ga} \mathrm{Cu}$ In and Mo. but gold is not in this paragenesis, suggesting that the paragenesis represent another sulphidation event that is barren with respect to $\mathrm{Au}$. The presence of $\mathrm{V}$ can be explained by its normal occurrence in small amount in magnetite [14]. The presence of $\mathrm{Cr}$ in this factor can be link by the presence of chromite in parent rocks. Scandium can be associated to organic matter. Its small size and high charge favor the formation of stable organic complexes in soils or absorption on clay minerals derived from the chemical weathering of granitic rocks [15]. The factor 1 spatial distribution (figure 3) show high levels in the SE of the study area on the Mayo Saboule river and in the NW on the Makoué river.

Factor 2: account for $26.90 \%$ of the total variability and is made up of the associations of $\mathrm{Ba}-\mathrm{Sr}-\mathrm{Ag}-\mathrm{Cr}-\mathrm{Cu}-\mathrm{Co}-\mathrm{Be}-\mathrm{Ni}-\mathrm{Y}-$ $\mathrm{Zn}-\mathrm{V}$. These associations can be interpreted to reflect a lithological control. It can be attributed to the catchment weathering, and disseminated sulfides such as chalcopyrite, pyrite, and sphalerite. Reference 16 showed that Fe can be substituted for by $\mathrm{Co}, \mathrm{Ni}$ in magnetite reflecting the receptivity of the octahedral and tetrahedral sites in spinel lattices; V can be explained by its normal occurrence in small amounts in magnetite [14].

Factor 3: comprise Ga-Hf-Zr and explains $13.04 \%$ of the total variance. Reference [17] proposed that the enrichment of any stream sediment in high field strength elements (HFSE) such as Hf-Zr is a strong indication of granitic source. This factor indicate a granitic origin, and present a high leadings in the SE of the study area on the Lawana river (figure 3).

Table 5. Percentage of variance and cumulative variance of factors.

\begin{tabular}{llll}
\hline \multirow{2}{*}{ Component } & \multicolumn{3}{l}{ Initial values } \\
\cline { 2 - 4 } & Total & \% of variance & \% of cumulative \\
\hline 1 & 12.93 & 39.20 & 39.20 \\
2 & 8.87 & 26.90 & 66.11 \\
3 & 4.30 & 13.04 & 79.15 \\
4 & 2.56 & 7.76 & 86.92 \\
5 & 2.13 & 6.47 & 93.40 \\
\hline
\end{tabular}

Factor 4: $\mathrm{Sn}-\mathrm{Au}$; this factor explains $7.7 \%$ of the total variance. This is a mineralization factor. Cassiterite deposit is known in the Adamawa region in granitic rocks context and associated veins. This factor shows that cassiterite deposit and gold mineralizations are contemporaneous. This factor present a high leadings in the center part of the study area (sample S504), and medium leadings on mayo Nangue, Mbikoum, Makoué, Mardock rivers (figure 3).

Factor 5: As-Cd explains about $6.47 \%$ of the total variability (table 5). It is also a mineralization factor which represents another sulphidation event that is barren with 
respect to Au. This factor is more express in the central part of the study area, on the Mayo Nangue river (figure 3).
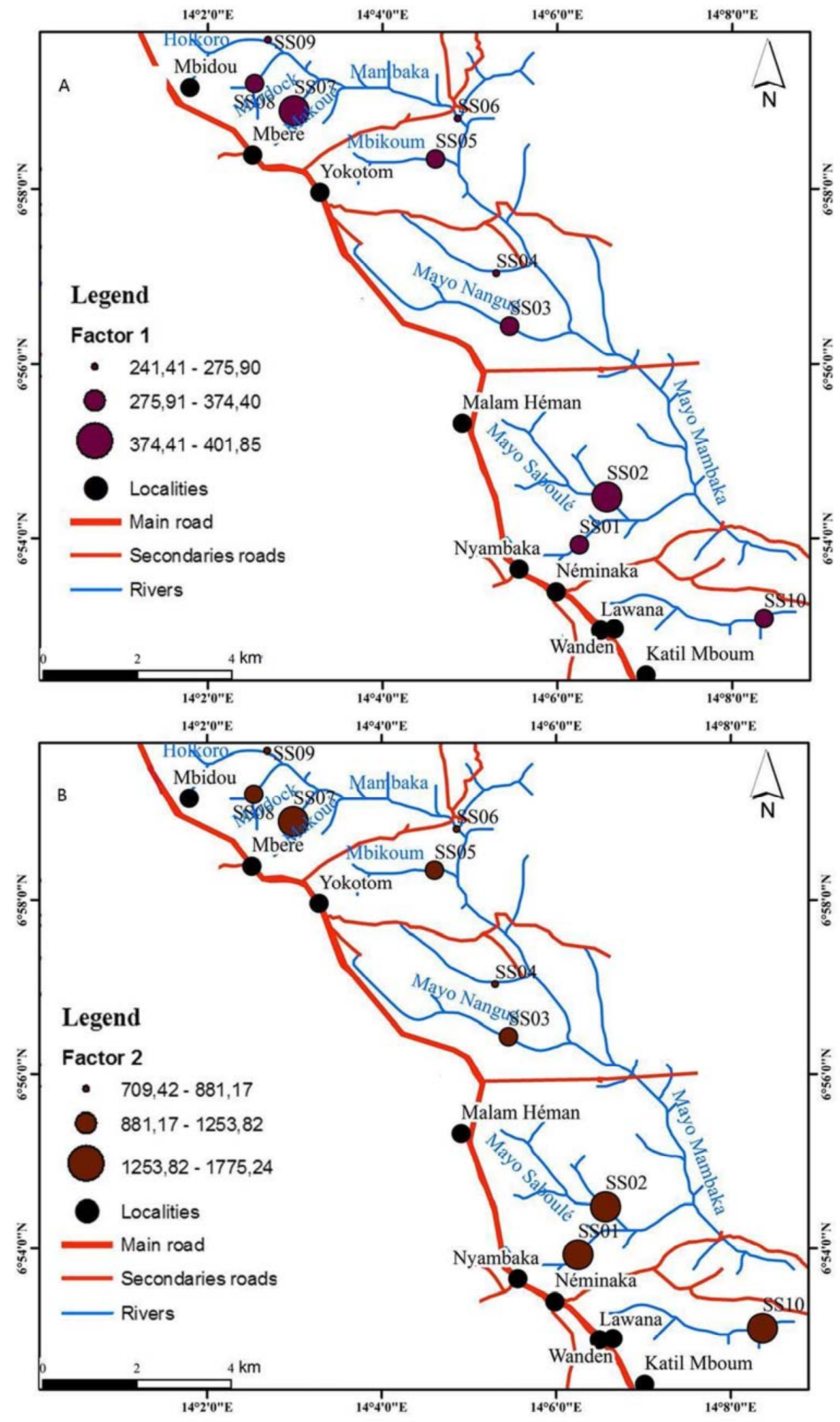


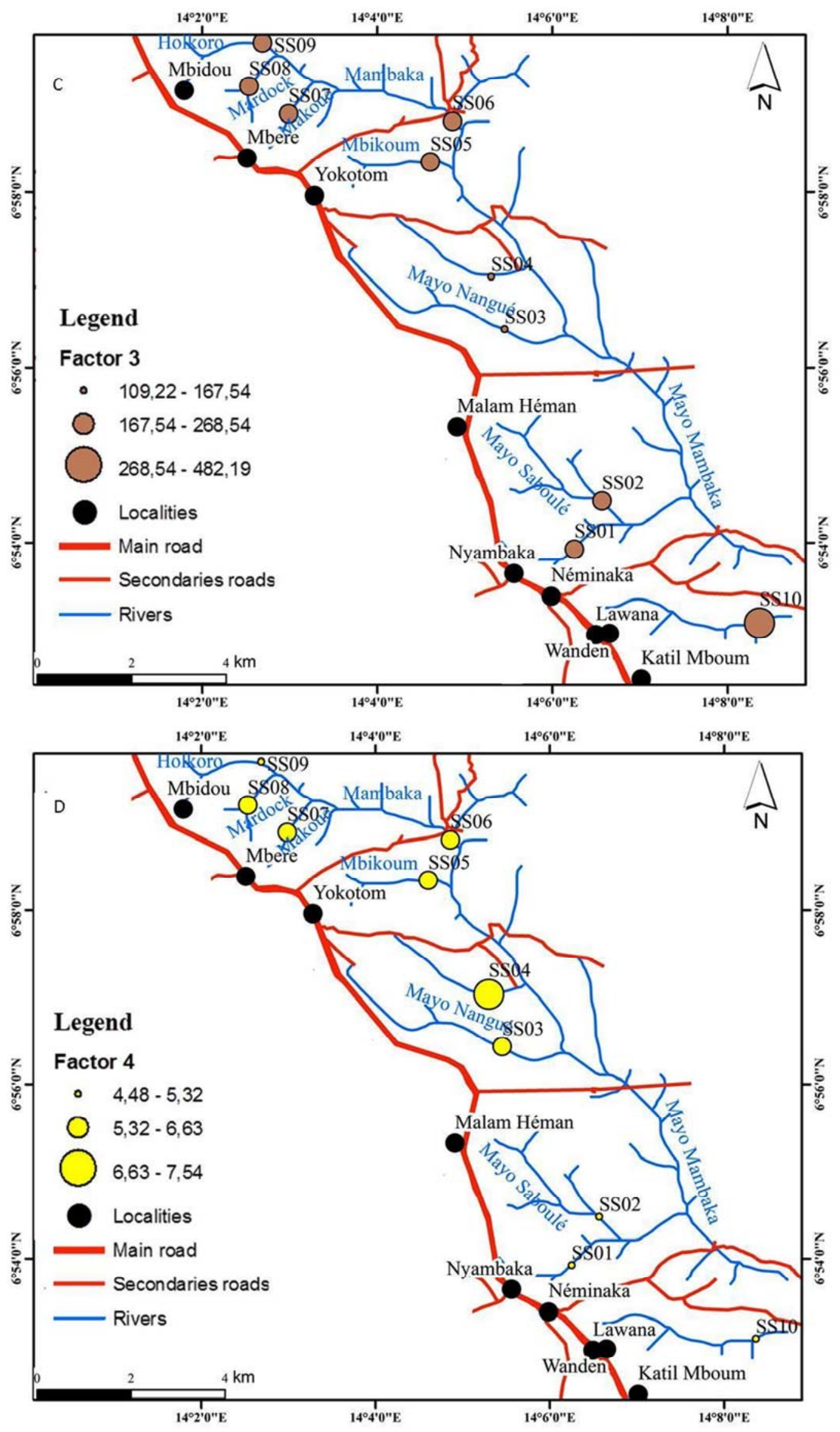




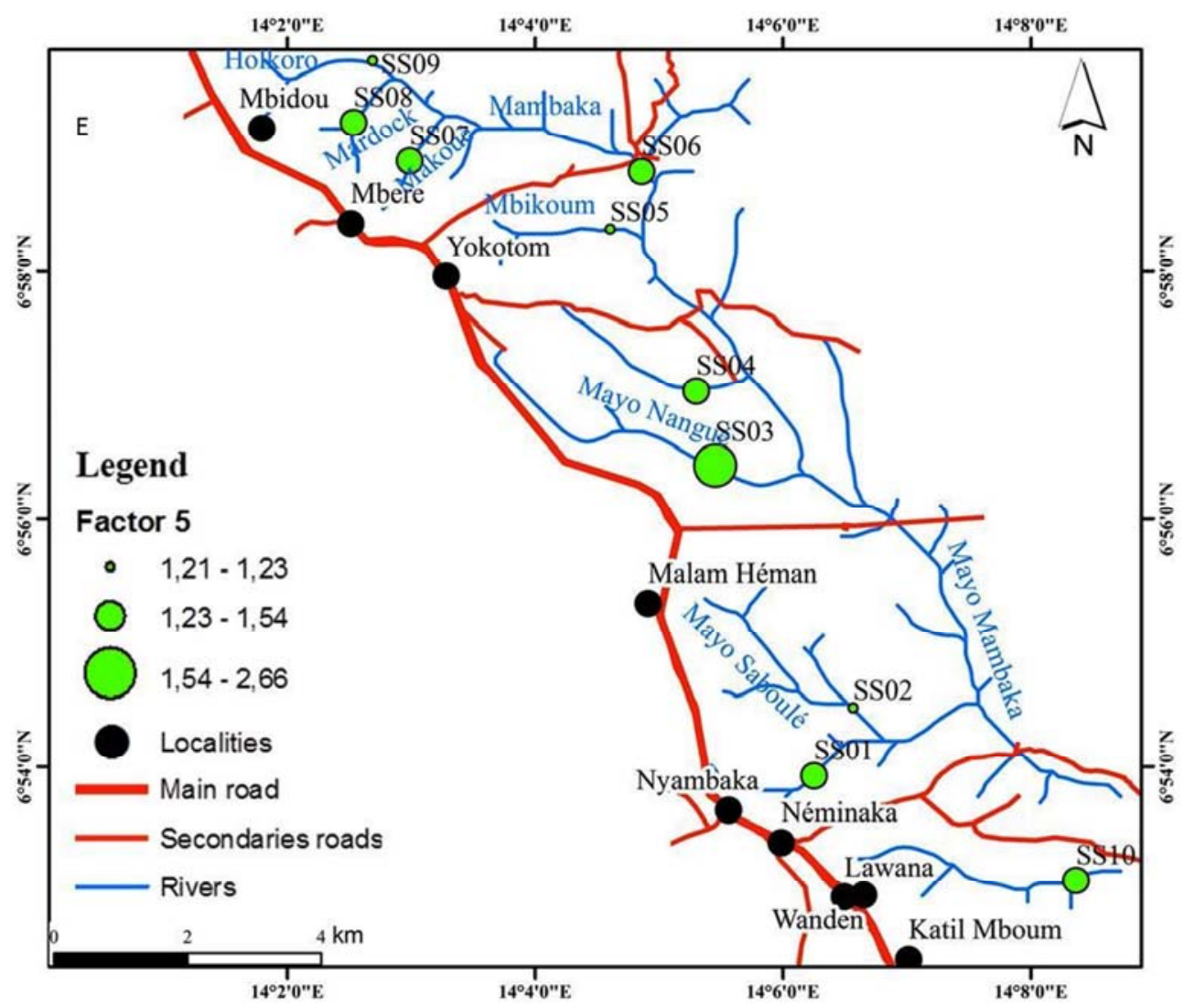

Figure 3. Spatial distribution of the factors over the watershed of the studied area.

\section{Conclusion}

As conclusion, we can say that:

Statistical and spatial analysis was carried out to relate the concentration and the distribution of chemical elements in stream sediments to lithology and mineralizations.

The factor analysis applied to the data defines five factors that explained about $93.2 \%$ of the total variance. Factor 1 accommodate elements Sc-Mo-V-In-Ga-Cu-Cr. this association reflect an hydrothermal alteration leading to the development of $\mathrm{Ga}$; $\mathrm{Cu}$, In and Mo. this suggest that the paragenesis represent another sulphidation event that is barren with respect to Au.
Factor 2 (Ba-Sr-Ag-Cr-Cu-Co-Be-Ni-Y-Zn-V) These associations can be attributed to the catchment weathering, and disseminated sulfides such as chalcopyrite, pyrite, and sphalerite.

Factor 3 (Ga-Hf-Zr) explains $13.04 \%$ of the total variance. This factor indicates a granitic origin.

Factor $4(\mathrm{Sn}-\mathrm{Au})$ explains $7.7 \%$ of the total variance and indicate that gold and cassiterite mineralizations are contemporaneous and link to granitic rocks context and associated veins.

Factor 5 (As-Cd) explains about $6.47 \%$, and also indicates another sulphidation event that is barren with respect to Au.

These results highlight the fact that gold mineralization is link to granitic rocks.

Table 6. Geochemical data of the concentration of elements in stream sediments from Nyambaka area.

\begin{tabular}{|c|c|c|c|c|c|c|c|c|c|c|c|c|}
\hline Elements & Unit & DL & SS01 & SS02 & SS03 & SS04 & SS05 & SS06 & SS07 & SS08 & SS09 & SS10 \\
\hline Mo & PPM & 0.01 & 1.81 & 1.71 & 1.93 & 1.95 & 1.86 & 1.50 & 1.86 & 2.06 & 1.42 & 1.62 \\
\hline $\mathrm{Cu}$ & PPM & 0.01 & 50.86 & 53.45 & 42.86 & 22.54 & 42.70 & 27.57 & 46.42 & 46.35 & 24.30 & 54.21 \\
\hline $\mathrm{Pb}$ & PPM & 0.01 & 5.81 & 6.00 & 8.27 & 7.56 & 8.52 & 12.49 & 7.77 & 6.75 & 13.31 & 5.96 \\
\hline $\mathrm{Zn}$ & PPM & 0.1 & 73.70 & 89.60 & 57.10 & 29.20 & 44.70 & 52.90 & 72.10 & 73.30 & 44.30 & 85.40 \\
\hline $\mathrm{Ag}$ & PPB & 2.00 & 24.00 & 23.00 & 21.00 & 16.00 & 22.00 & 22.00 & 18.00 & 23.00 & 15.00 & 21.00 \\
\hline $\mathrm{Ni}$ & PPM & 0.1 & 170.70 & 231.10 & 110.50 & 36.40 & 108.40 & 86.20 & 160.90 & 162.50 & 78.20 & 198.00 \\
\hline $\mathrm{Co}$ & PPM & 0.1 & 45.20 & 63.80 & 21.70 & 12.90 & 34.60 & 41.10 & 26.40 & 47.10 & 23.40 & 65.70 \\
\hline $\mathrm{U}$ & PPM & 0.1 & 2.30 & 2.20 & 2.70 & 2.20 & 2.80 & 3.70 & 2.40 & 2.20 & 4.00 & 2.40 \\
\hline $\mathrm{Au}$ & PPB & 0.2 & 0.70 & 0.40 & 1.00 & 0.60 & 1.00 & 0.70 & 0.90 & 0.90 & 0.70 & 1.00 \\
\hline Th & PPM & 0.1 & 12.30 & 12.50 & 13.10 & 9.80 & 16.70 & 20.30 & 14.60 & 13.60 & 25.60 & 13.40 \\
\hline $\mathrm{Sr}$ & PPM & 0.5 & 38.20 & 39.10 & 14.50 & 7.10 & 20.10 & 34.40 & 16.90 & 28.80 & 19.50 & 105.00 \\
\hline $\mathrm{Cd}$ & PPM & 0.01 & 0.03 & 0.03 & 0.06 & 0.03 & 0.01 & 0.04 & 0.04 & 0.04 & 0.02 & 0.06 \\
\hline $\mathrm{Sb}$ & PPM & 0.02 & 0.00 & 0.00 & 0.06 & 0.06 & 0.04 & 0.03 & 0.04 & 0.03 & 0.02 & 0.00 \\
\hline $\mathrm{Bi}$ & PPM & 0.02 & 0.03 & 0.00 & 0.07 & 0.07 & 0.08 & 0.06 & 0.05 & 0.04 & 0.06 & 0.02 \\
\hline
\end{tabular}




\begin{tabular}{|c|c|c|c|c|c|c|c|c|c|c|c|c|}
\hline Elements & Unit & DL & SS01 & SS02 & SS03 & SS04 & SS05 & SS06 & SS07 & SS08 & SS09 & SS10 \\
\hline V & PPM & 1 & 266.00 & 312.00 & 277.00 & 197.00 & 271.00 & 168.00 & 308.00 & 269.00 & 142.00 & 267.00 \\
\hline $\mathrm{Ca}$ & $\%$ & 0.01 & 0.08 & 0.05 & 0.02 & 0.00 & 0.04 & 0.14 & 0.02 & 0.06 & 0.07 & 0.25 \\
\hline $\mathrm{Cr}$ & PPM & 0.5 & 548.10 & 763.00 & 393.80 & 273.30 & 487.00 & 221.90 & 614.70 & 412.80 & 202.70 & 514.70 \\
\hline $\mathrm{Ba}$ & PPM & 0.5 & 137.70 & 151.50 & 73.40 & 43.20 & 83.40 & 171.90 & 100.60 & 131.80 & 125.80 & 325.20 \\
\hline $\mathrm{Sc}$ & PPM & 0.1 & 31.70 & 31.20 & 29.70 & 24.90 & 34.50 & 20.00 & 33.40 & 29.50 & 15.20 & 32.20 \\
\hline $\mathrm{Tl}$ & PPM & 0.02 & 0.07 & 0.07 & 0.08 & 0.07 & 0.08 & 0.22 & 0.05 & 0.07 & 0.22 & 0.06 \\
\hline $\mathrm{Hg}$ & PPB & 5.00 & 29.00 & 18.00 & 32.00 & 54.00 & 18.00 & 24.00 & 17.00 & 27.00 & 19.00 & 26.00 \\
\hline $\mathrm{Ga}$ & PPM & 0.1 & 26.60 & 25.40 & 31.90 & 28.80 & 34.60 & 23.80 & 30.70 & 28.40 & 20.40 & 24.40 \\
\hline Cs & PPM & 0.02 & 0.51 & 0.44 & 0.84 & 0.81 & 0.89 & 1.12 & 0.53 & 0.53 & 1.08 & 0.58 \\
\hline $\mathrm{Hf}$ & PPM & 0.02 & 0.44 & 0.66 & 0.72 & 0.42 & 0.78 & 0.6 & 0.89 & 0.82 & 0.42 & 0.34 \\
\hline $\mathrm{Nb}$ & PPM & 0.02 & 1.41 & 1.28 & 2.43 & 4.04 & 0.91 & 2.67 & 1.69 & 2.21 & 1.52 & 1.05 \\
\hline $\mathrm{Rb}$ & PPM & 0.1 & 3.50 & 2.60 & 3.30 & 2.70 & 3.30 & 17.10 & 2.40 & 2.30 & 18.10 & 4.10 \\
\hline $\mathrm{Sn}$ & PPM & 0.1 & 3.00 & 2.80 & 3.20 & 2.90 & 3.80 & 2.80 & 3.20 & 3.10 & 3.10 & 2.90 \\
\hline $\mathrm{Zr}$ & PPM & 0.1 & 38.10 & 47.20 & 47.00 & 29.70 & 52.90 & 37.70 & 59.00 & 61.50 & 28.50 & 27.00 \\
\hline In & PPM & 0.02 & 0.14 & 0.12 & 0.13 & 0.11 & 0.15 & 0.10 & 0.14 & 0.13 & 0.08 & 0.12 \\
\hline $\mathrm{Be}$ & PPM & 0.1 & 2.20 & 2.60 & 2.30 & 0.60 & 1.20 & 1.80 & 2.00 & 2.00 & 1.90 & 2.00 \\
\hline
\end{tabular}

\section{Acknowledgements}

This study has been supported by Johnny Yao, M. Edima Nathanael, Oyono Amelie Majolie, Ngbwa Emile, Bureau Veritas commodities Canada LTD and the population of the Nyambaka District.

\section{References}

[1] Mumbfu E. M., Takeshi O., Nguemhe Fils S. C., Tamnta Nforba M., Numanami N., Tasin G. B., Tongwa Aka F., Suh C. E., 2018. Regional geochemical baseline concentration of potentially toxic trace metals in the mineralized Lom Basin, East Cameroon: a tool for contamination assessment. Geochem Trans 19: 11. https://doi.org/10.1186/s12932-0180056-5.

[2] Mimba ME, Nforba MT, Suh CE, 2014. Geochemical dispersion of gold in stream sediments of the Paleoproterozoic Nyong Series, southern Cameroon. Sci Res 2 (6): 155-165.

[3] Embui VF, Omang BO, Che VB, Nforba MT, Suh EC, 2013. Gold grade variation and stream sediment geochemistry of the theVaimba-Lidi drainage system, northern Cameroon. Nat Sci 5 (2A): $282-290$

[4] Key, R. M., De Waele, B., Liyungu, A. K., 2004. A multielement baseline geochemical database from the western extension of the Central Africa Copperbelt in northwestern Zambia. Transactions of the Institution of Mining and Metallurgy. Section B: Applied Earth Science, 113, B205B226. doi: 10.1179/037174504225005717.

[5] Salminen, R., Kashabano, J., Myumbilwa, Y., Petro, F. N. and Partanen, M., 2008. Indications of deposits of gold and platinum group elements from a regional geochemical stream sediment survey in NW Tanzania. Geochemistry: Exploration, Environment Analysis, 8, 313-322. doi: 10.1144/14677873/08-179.

[6] Penaye, J., Toteu, S. F., Tchameni, R., Van Schmus, R. W., Tchakounté, J., Ganwa, A. A., Nsifa, N. E., Minyem, D., 2004. The $2.1 \mathrm{Ga}$ West Central African belt in Cameroon: extension and evolution. J. Afr. Earth Sci. 39, $159-164$.
[7] Tchaméni, R., Pouclet, A., Penaye, J., Ganwa, A. A., Toteu, S. F., 2006. Petrography and geochemistry of the Ngaoundéré Pan-African granitoids in Central North Cameroon: Implications for sources and geological setting. J. Afr. Earth Sci. 44, 511-529.

[8] Ganwa, A. A., Frisch, W., Siebel, W., Shang, C. K., Mvondo Ondoa, J., Tchakounté Numbem, J., 2008. Zircon ${ }^{207} \mathrm{~Pb} /{ }^{206} \mathrm{~Pb}$ evaporation ages of Pan-African metasedimentary rocks in the Kombé-II area (Bafia Group, Cameroon): Constraints on protolith age and provenance. J. Afr. Earth Sci. $51,77-88 .{ }^{207} \mathrm{~Pb} /{ }^{206} \mathrm{~Pb}$ evaporation ages of Pan-African metasedimentary rocks in the Kombé-II area (Bafia Group, Cameroon): Constraints on protolith age and provenance. J. Afr. Earth Sci. 51, 77-88.

[9] Nkouandou O. F., Ngounouno I., Deruelle B., 2010. Géochimie des laves basaltiques récentes des zones Nord et Est de Ngaoundéré (Cameroun, Plateau de l'Adamaoua, Afrique Centrale): pétrogenèse et nature de la source. Int. J. Biol. Chem. Sci. 4(4), 984-1003.

[10] Van Schmus, W. R., Oliveira, E. P., Da Silva Filho, A. F., Toteu, S. F., Penaye, J., Guimarães, I. P., 2008. Proterozoic links between the Borborema Province, NE Brazil, and the Central African Fold Belt. In: Pankhurst, R. J., Trouw, R. A. J., Brito Neves, B. B., De Wit, M. J. (Eds), West Gondwana: PreCenozoic Correlations Across the South Atlantic Region. Geol. Soc. London Spec. Publ. 294, 69-99.

[11] Toteu, S. F., Van Schmus, R. W., Penaye, J., Michard, A., 2001. New U-Pb and Sm- Nd data from north-central Cameroon and its bearing on the pre-Pan-African history of central Africa. Precambrian Res. 108, 45-73.

[12] Ganwa, A. A., Klötzli, U.S., Hauzenberger, C., 2016. Evidence for Archean inheritancein the pre-Panafrican crust of Central Cameroon: Insight from zircon internal structure and LA-MC-ICP-MS U-Pb ages. J. Afr. Earth Sci. 120, 12-22.

[13] J. Tchakounté, A. Eglinger, S. F. Toteu, A. Zeh, C. Nkoumbou, J. Mvondo-Ondoa, J. Penaye, Martin de Wit, P. Barbey, 2017. The Adamawa-Yadé domain, a piece of Archaean crust in the Neoproterozoic Central African Orogenic belt (Bafia area, Cameroon). Precambrian Research 299 210-229.

[14] Kessler, W., Müller, 1988. Minor and Trace-element data of iron oxides from iron-formations of the Iron Quadrangle, Minas Gerais, Brazil. Mineralogy and Petrology 39, 245250. 
[15] Yang XJ, Lin A, Li XL, Wu Y, Zhou W, Chen Z, 2013. China's ion-adsorption rare earth resources, mining consequences and preservation. Environ Dev 8: 131-136. https://doi.org/10.1016/j.envdev.2013.03.006.

[16] Surour, A. A., El-Kammar, A. A., Arafa, E. H., Korany, H. M., 2003. Dahab Stream Sediments southeastern Sinai, Egypt: a potential source of gold, magnetite and zircon. Journal of Geochemical Exploration 7 (1), 25-43.

[17] Chandrajith R, Dissanayake CB, Tobshall HJ (2001) Enrichment of high field strength elements in stream sediments of a granulite terrain in Sri Lanka-evidence for a mineralised belt. Chem Geol 175: 259-271. 